\title{
Control of waste fragment sorting process based on MIR imaging coupled with cautious classification
}

\author{
Lucie Jacquin *, , Abdelhak Imoussaten ${ }^{\mathrm{a}}$, François Trousset ${ }^{\mathrm{b}}$, Didier Perrin ${ }^{\mathrm{c}}$, Jacky Montmain ${ }^{\mathrm{a}}$ \\ ${ }^{\text {a }}$ EuroMov Digital Health in Motion, Univ Montpellier, IMT Mines Ales, France \\ ${ }^{\mathrm{b}}$ Laboratoire des Sciences des Risques (LSR), IMT Mines Ales, France \\ ${ }^{\mathrm{c}}$ Polymers Composites and Hybrids (PCH), IMT Mines Ales, France
}

A B S T R A C T

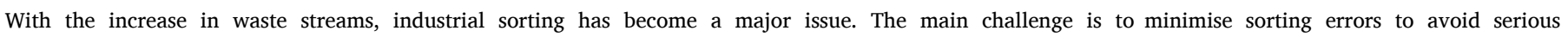

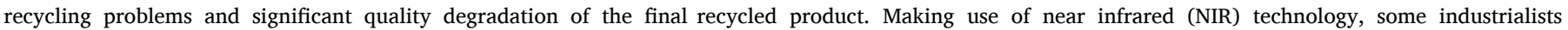

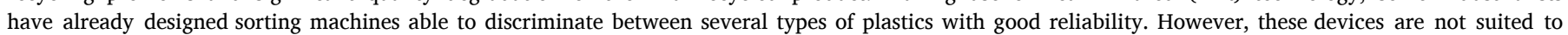

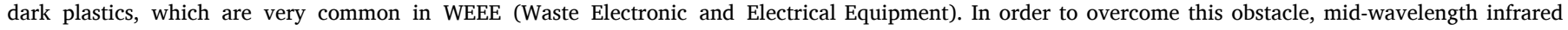

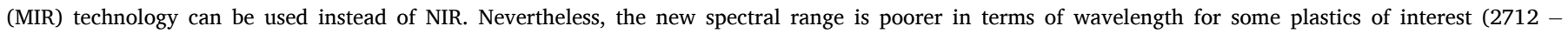

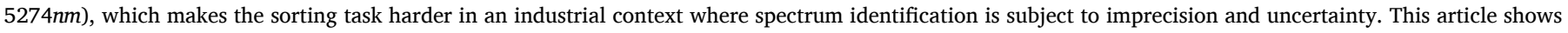

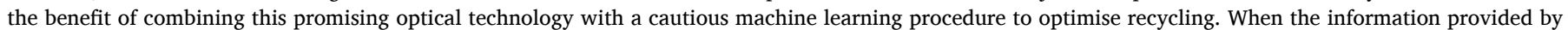

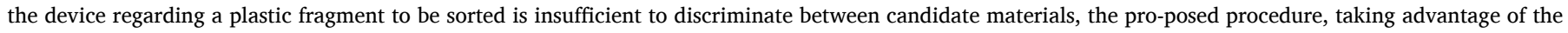

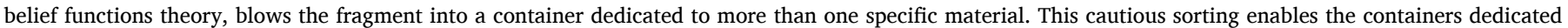

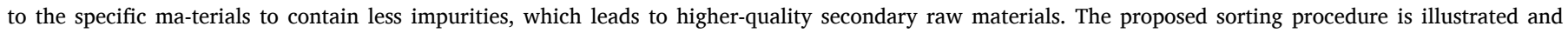
compared with a more conventional approach using real industrial data.

\section{Introduction: context and challenges}

The mass production of technologies with planned obsolescence and little concern for ecodesign has caused the emergence of one of the fastest growing waste streams worldwide (Cucchiella et al., 2015), Waste Electrical and Electronic Equipment (WEEE). Indeed, estimations of the growth rate of WEEE range from $3 \%$ to $5 \%$ per year (Cucchiella et al., 2015). Moreover, the disposal of WEEE is complex as it contains numerous hazardous materials that involve health risks (Matarazzo et al., 2019). Because of these growing environmental and sanitary issues, WEEE is receiving special attention from the authorities. The European Commission strengthened the minimum recovery targets of WEEE in Directive 2012/19/EU (eu1, 2012), according to which, for 10 categories of WEEE, at least $80 \%$ should be prepared for re-use and recycling. In order to comply with this regulation, technological advances in the recycling process are required. In this process, a major phase is the separation of materials, which directly impacts all further processing and consequently the final quality of recycled products. Separation needs to be performed with high reliability and massive capacity to cope with the huge amount of WEEE. This article focuses on the stream of plastics, which represent $\sim 21 \%$ by weight of the materials found in WEEE (Ongondo et al., 2011). The sorting of these plastics is becoming increasingly complex because of the wide variety of existing types of plastics that share similar visual characteristics but are usually incompatible. The separation of these plastics is consequently carried out a posteriori, i.e. all plastics are collected together, before being shredded and sorted. To ensure the high reliability and massive capacity of the sorting process in such a configuration, i.e. considerable quantities of plastics of very different kinds to be sorted in a very short time, many industrialists are considering on-line optical sorting devices.

The optical sorting technologies that are already widespread in the food industry are proving to be highly appropriate for sorting plastic waste. Compared to manual sorting, they offer significant advantages such as better throughput and more competitive labour costs. For sorting

\footnotetext{
* Corresponding author.

E-mail address: lucie.jacquin@mines-ales.fr (L. Jacquin).
} 
plastics, infrared processes are the appropriate optical technology since they can quickly collect information about groups of atoms on the surface. The spectral information (coming from absorption or transmittance measurements) used by industrialists to discriminate between plastics is mostly derived from the NIR and MIR spectrum ranges.

Making use of NIR technology (wavelengths: $700-1400 \mathrm{~nm}$ ), some industrialists have designed sorting machines able to discriminate between several types of plastics. However, the use of these machines is not suited to dark plastics, which are very common in WEEE. The reason for this limitation is that dark and black plastics contain carbon black, which absorbs such a high proportion of NIR that it makes the plastic signature unrecognisable. In order to overcome this obstacle, MIR technology (wavelengths: $3000-8000 \mathrm{~nm}$ ) is a promising alternative to NIR. The impact of carbon black on the spectral signature is far less problematic at higher wavelengths. MIR technology has already been the subject of investigation. For example, Rozenstein et al. (2017) identified MIR absorption features for several black plastics. In addition, Kassouf et al. (2014) presented a feasibility study for discriminating between virgin PE, PET, PP, PS and PLA, including some black samples, using Attenuated Total Reflectance (ATR) to characterize them. Other technologies such as THz, LIBS and Raman are alternative potential solutions for the treatment of black plastics. However, THz does not seem mature enough to be incorporated in an industrial device and LIBS is limited by the restricted number of chemical elements that it can measure. Concerning Raman, a comparative study of ATR FT-IR and Raman spectroscopy suggested that Raman technology can also produce highly competitive results for discriminating black plastic waste (Bae et al., 2019). However, due to the low intensity of its rays which is much lower than Rayleigh rays, it is difficult to provide competitive online acquisition using Raman technology.

MIR is therefore the technique most seriously envisaged by industrialists for sorting plastics coming from WEEE. When using a plastic identification technology in an industrial context, the quality of acquired data may be significantly affected in several ways, which will be explained below. The challenge addressed in this article is thus how to process these imperfect data to ensure the most reliable possible sorting results. In practice, the principle of on-line plastic sorting is as follows. Fragments of plastics or objects are delivered continuously on a conveyor belt before being filmed by a MIR camera. For each fragment to be sorted, the camera provides a set of spectra where each spectrum corresponds to a pixel in the hyperspectral image of the fragment. These spectra are then processed by an integrated algorithm in order to determine the most appropriate container in which to blow the fragment. Fig. 1, taken from Beigbeder et al. (2013) illustrates such a device. To the best of our knowledge, the existing integrated algorithms often used in industry perform voting to merge information about each fragment pixel when sorting fragments based on hyperspectral imaging (Lorente et al., 2012; Blanch-Perez-del Notario et al., 2019; Paclik and Duin, 2005; Paclik et al., 2006). In this case each pixel is assigned a plastic type (classification phase). Afterwards, a majority vote procedure

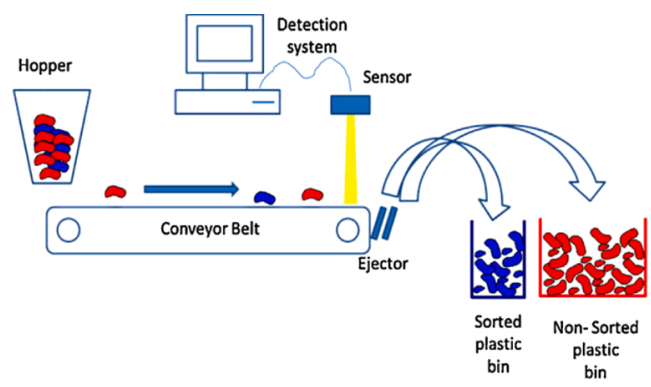

Fig. 1. Example of a sorting device (Beigbeder et al., 2013). on the pixel types enables a type to be assigned to the fragment. Finally the fragment is blown into the container corresponding to its type (sorting phase). Dealing with imperfect data, these conventional approaches face two different problems that could affect sorting performances.

The first problem (1) concerns the absence of any adequate representation of data imperfection. Consequently imperfect data could be mishandled and cause sorting errors. The second problem (2) concerns the output. The fragments are systemically assigned a single type even when the information is insufficient to make a decision. Hence, all imperfectly measured fragments will still be blown into containers dedicated to single plastic types, inevitably leading to sorting errors. This second problem is highly related to the first because the representation of the information impacts the output: if imprecision and uncertainty are not represented, they cannot be taken into account to achieve a more cautious output.

Problem (1) causes us to consider alternative representations of the information that take into account imprecision and uncertainty. For this purpose, information could be retained about posterior probabilities of each type being the true type of a given fragment. However, posterior probabilities are unable to represent imprecision because of the inherent additivity constraint of probabilities. For example, consider a fragment for which the device provides information such that none of the plastic types could correspond to the spectral information. In such a case, the probabilities that the candidate plastic types could be the real type of the fragment are wrongly inflated to sum to 1 . Any insignificant resemblance to one of the types would then determine the fragment sorting. In order better to represent the imperfection of the information, modern theories of uncertainty such as fuzzy subsets (Zadeh, 1978), possibility theory (Dubois and Prade, 2012), imprecise probabilities (Walley, 2000) or belief functions (Shafer, 1976; Smets and Kennes, 1994) are used instead of probability theory. Several classification algorithms have been proposed in these frameworks. Most of them are extensions of standard algorithms. One can cite the fuzzy version of the well known $k$-means algorithm (Dunn, 1973), fuzzy and belief functions based versions of $k$-Nearest Neighbour ( $k$-NN) (Denoeux, 1995; Keller et al., 1985) or even some fuzzy and belief function based revisions of neural networks algorithms (Denoeux, 2000; Kruse, 2008). Such theories of uncertainty have already been used in the context of plastic sorting. For example, Lachaize et al. (2018) used belief function theory to perform segmentation and material class estimation based on RGB images, laser profilometer and NIR imaging. Alternatively, S.-B. Roh et al. (2017) applied a fuzzy radial basis function neural network classifier to identify black plastics based on Raman spectra. The present article focuses on the belief function theory framework because it is designed to express imprecision and uncertainty and offers a wide range of options for combining information. The latter point is a necessary step for merging the information coming from all the pixels of a fragment.

Regarding problem (2), the proposition of this paper is to consider more cautious sorting options when the information concerning some fragments is insufficient to assign a single material-type to them. The cautious option would be to blow such fragments into new containers dedicated to more than one single material. In order to operate this cautious sorting, the proposed approach performs cautious classification by allowing partial abstention for difficult fragments, i.e. associating a set of candidate types to a fragment instead of a single type. As explained in Nguyen et al. (2018), cautious classification was introduced by the idea of abstention, which was proposed by Chow (1970) in the 1970s with the notion of the rejection option. However, partial abstention in the form of a subset of classes is more recent (Ha, 1997). Although cautious classification can increase the reliability of the algorithm output, it also needs to be relevant enough, i.e. the set of candidate types must be as small as possible. The aim of this paper is to show that appropriate management of uncertainty combined with a cautious sorting option can be advantageous for on-line sorting, here applied to dark and black plastics. For this purpose the presented approach 


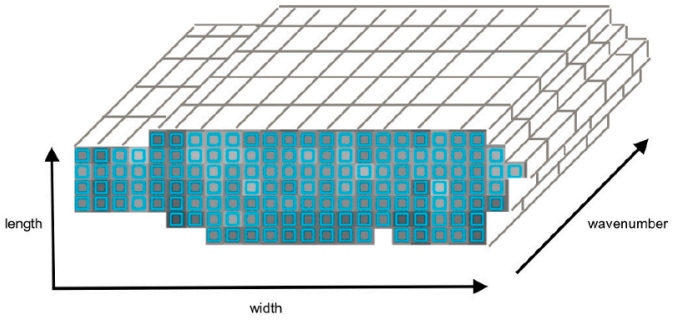

Fig. 2. 3D refection image of a plastic fragment.

performs cautious sorting of fragments based on belief functions and MIR imaging. Our study focuses more particularly on the sorting of specific styrenic and polyolefin polymers that are valuable for industrial purposes and found as majority-materials in several studies concerning WEEE (Signoret et al., 2019b).

\section{Materials and methods}

\subsection{Types of materials and plastics supplied}

The types of materials and plastics considered in this article are the four types of petro-based polymer waste: acrylonitrile-butadiene-styrene $(A B S)$, high impact polystyrene (HIPS), polyethylene (PE) and polypropylene $(P P)$. The data considered in this study concerned 280 plastic fragments originating from the Suez firm and comprising dark, black and coloured plastics. To ensure sufficient storage density, the size of the fragments was rather small, approximately $2 \times 2 \mathrm{~cm}^{2}$. Moreover, each plastic fragment was labelled based on trustworthy expert identifications from ATR spectra obtained from a common FT-IR apparatus.

\subsection{MIR spectral acquisition}

This section presents the industrially acquired information concerning the plastic fragments considered in this study. A Specim MIR camera was used to record the information concerning the plastic fragments conveyed at speed $2 \mathrm{~m} \cdot \mathrm{s}^{-1}$ (accumulation time equal to $1.3 \mathrm{~ms}$ and single cycle of detection). The source used to illuminated the fragments is a focused Pellenc ${ }^{1}$ source, emitting according to a thermal spectrum (blackbody law), with a temperature optimised to maximise the signal in the $3000-5000 \mathrm{~nm}$ range. The speed $2 \mathrm{~m}^{-1} \mathrm{~s}^{-1}$ permits to preserve sufficient quality of the acquired information while remaining competitive. Fig. 2 represents the 3D reflection image acquired by the camera for a given fragment. The two first dimensions indicate the pixel's location on the width and length of the conveyor. The third dimension of the image corresponds to the wavelength/wavenumber value, i.e. one of the 154 values distributed equidistantly on 2712 $5274 \mathrm{~nm}$ (corresponding to not equidistant points on 3687 $1896 \mathrm{~cm}^{-1}$ ). Some pre-processing was applied to these data, for example in order to reduce light scattering and spectral noise effects, all the pixels' spectra were corrected by the Standard Normal Variate (SNV) technique.

Fig. 3 illustrates some spectra (corresponding to pixels of several fragments) recorded by the camera for the four types of plastics (coloured curves) compared with the corresponding ATR reference spectra (black curve) on an arbitrary y-axis scale. Note that the peaks of

\footnotetext{
${ }^{1}$ Pellenc Selective Technologies (or Pellenc ST) is a company that designs, produces and markets intelligent and connected sorting equipment and services for the recycling industry.
}

reflectance spectra are downward while the ATR spectra peaks are upward. On the same figure, the vertical dashed lines show the special blends that are the most intense in ATR (see Signoret et al., 2019a; Signoret et al., 2019c for more details). The ATR curve was obtained under ideal conditions (static and in contact with the sample) so the peaks are very clear and it contains perfect information for plastic identification. On the other hand, the reflectance spectra recorded in dynamic mode by the MIR camera contain poorer information. In particular, the acquired information is subject to several issues inducing the presence of imprecision, i.e. some information features are not precise enough to draw clear distinctions between the material-types, and uncertainty, i.e. some obtained features are not completely consistent with the reality. The sources of imprecision or uncertainty are as follows i) the available spectral range might be insufficient, it is only $1896-3687 \mathrm{~cm}^{-1}$ for the industrial spectra while the range $400-$ $4000 \mathrm{~cm}^{-1}$ is available for the ATR spectra; ii) the plastic types to be recycled are chemically close, and therefore spectrally close, for example $A B S$ and HIPS can theoretically only be distinguished by the acrylonitrile peak at $2236 \mathrm{~cm}^{-1}$; iii) atmospheric disturbance may cause noise, which explains some of the wide variance in spectra for the same materials in Fig. 3; iv) plastic ageing and plastic additives are known to change spectral information; v) impurities like dust deposits or the remains of tags will also produce spectral noise, etc.

For simplification purposes, in the rest of this paper a spectrum vector comprised of its 154 reflectance values is referred to as a pixel.

\subsection{Proposed approach}

In this section, the proposed cautious sorting approach is presented. The objective of this approach is to optimise recycling by minimising the impurities in the resulting containers when the available information is imperfect. The proposed approach consists in training a cautious classifier for the task of recognising fragments of material-types ABS, HIPS, $P E$ or $P P$ and then applying it to the sorting procedure. In the rest of this paper, $\Omega$ refers to the whole set of material-types $\{A B S, H I P S, P E, P P\}$. For a new fragment $f r$ the classifier is able to provide cautious information concerning the true material-type $\omega \in \Omega$ of $f r$. The simplified form of the cautious information is " $\omega \in A$ " where A is a subset of $\Omega$, meaning that each single material-type in $A$ is a candidate for the true material-type $\omega$ and the missing information prevents a more precise output. The set $A$ provided by the cautious classifier for $f r$ is intended to contain the real material-type of $f r$, but also to be as small as possible. In other words, the objective of a cautious classifier is to be reliable for any quality of information, i.e. A should always contain $\omega$, and be relevant, i.e. give a precise output, when the information is sufficient for a decision to be made. The following subsections present in more detail how the proposed procedure performs cautious classification of a fragment $f r$ composed of $k$ pixels, denoted $s_{1}, \cdots, s_{k}$. First, the information provided by a cautious classifier, about the material-type of each pixel of $f r$ is represented in the framework of belief functions. Then the information is merged to obtain the information about the material-type of $f r$. Finally, the cautious output for $f r$ is performed based on risk minimisation. The outline of the procedure is represented in Fig. 4 on an example of a single plastic fragment composed by six pixels (the representation pattern of pixels is the same as in Fig. 2 except that the fragment image is shown from the front and not in perspective). Finally, subsection 2.5 presents some evaluation measures for cautious classifiers.

\subsubsection{Pixel classification}

The first step is to collect and represent information about all pixels in the belief function framework proposed by Dempster (1967) and developed by Shafer (1976). This framework is a generalization of the probability framework that assigns mass probabilities to subsets of plastics. A classical probabilistic classifier provides a posterior 
probability $p(. \mid f r)$ on $\Omega$ as output. In our application a probabilistic classifier would return a probability for each material to be the true material-type $\omega$ of $f r$, namely $p(A B S \mid f r), p(H I P S \mid f r), p(P E \mid f r)$ and $p(P P \mid f r)$. For example, having $p(A B S \mid f r)=0.8$ means that the probability that $\omega$ is $A B S$ is 0.8 . Some classifiers can provide more general representation of uncertainty and imprecision in the data in the form of a mass function. A mass function $m$ is a probability mass function (discrete probability) defined on all the possible subsets of $\Omega$. A mass function can measure the mass exactly assigned to each subsets (excluding the mass provided by its elements). In other words, this framework assumes that one can "observe" a subset independently from the elements it contains, i.e. which corresponds to an imprecise piece of information.

Example 1. For example consider a pixel having spectral information on $2800-3300 \mathrm{~cm}^{-1}$ clearly assignable to $A B S$ or HIPS but such that the acrylonitrile peak at $2236 \mathrm{~cm}^{-1}$ (theoretically present for $A B S$ and absent for HIPS) is highly affected by imprecision. For such a pixel, there is a lack of information which prevents discrimination between $A B S$ and HIPS thus it is considered as $\{$ ABS, HIPS $\}$ material, i.e. Styrenic.

In the following paragraphs, the notation $m(. \mid x)$ refers to the posterior mass function representing the knowledge about the material-type of a pixel or fragment $x$. For example, $m(\{H I P S\} \mid f r)=0.4$ and $m(\{A B S$,
HIPS $\} \mid f r)=0.6$ means that the mass, i.e, the degree of belief, of the event "the true plastic type of fr is HIPS" is 0.4 and that the mass of the event "the true plastic type of $f r$ is $A B S$ or HIPS" is 0.6 . The subsets that are assigned non null masses are referred to as focal elements. In the previous example, the 2 focal elements were: $\{H I P S\}$ and $\{A B S, H I P S\}$. There are many classification algorithms able to return mass functions but they do not always consider the same focal elements:

- For some classification algorithms, all focal elements are singletons, here $\{A B S\},\{H I P S\},\{P E\}$ or $\{P P\}$. This actually amounts to considering a classical probability mass function (pms), which is a particular case of mass function where masses are only assigned to singletons ( $m(A \mid f r)=0$, where $A$ is a set comprising more than one element), i.e. no imprecision is considered. Classifiers outputting a pms are referred to as probabilistic classifiers.

- Some other classification algorithms consider singletons and the whole set $\Omega$ as focal elements. Unlike probabilistic classifiers, these classification algorithms are able to express their ignorance, represented by a mass assigned to the whole set $\Omega$. One of the best-known classification algorithms outputting such mass functions is the belief function-based version of $k$-nearest neighbour classification (Denoeux, 2008) proposed by Denoeux.
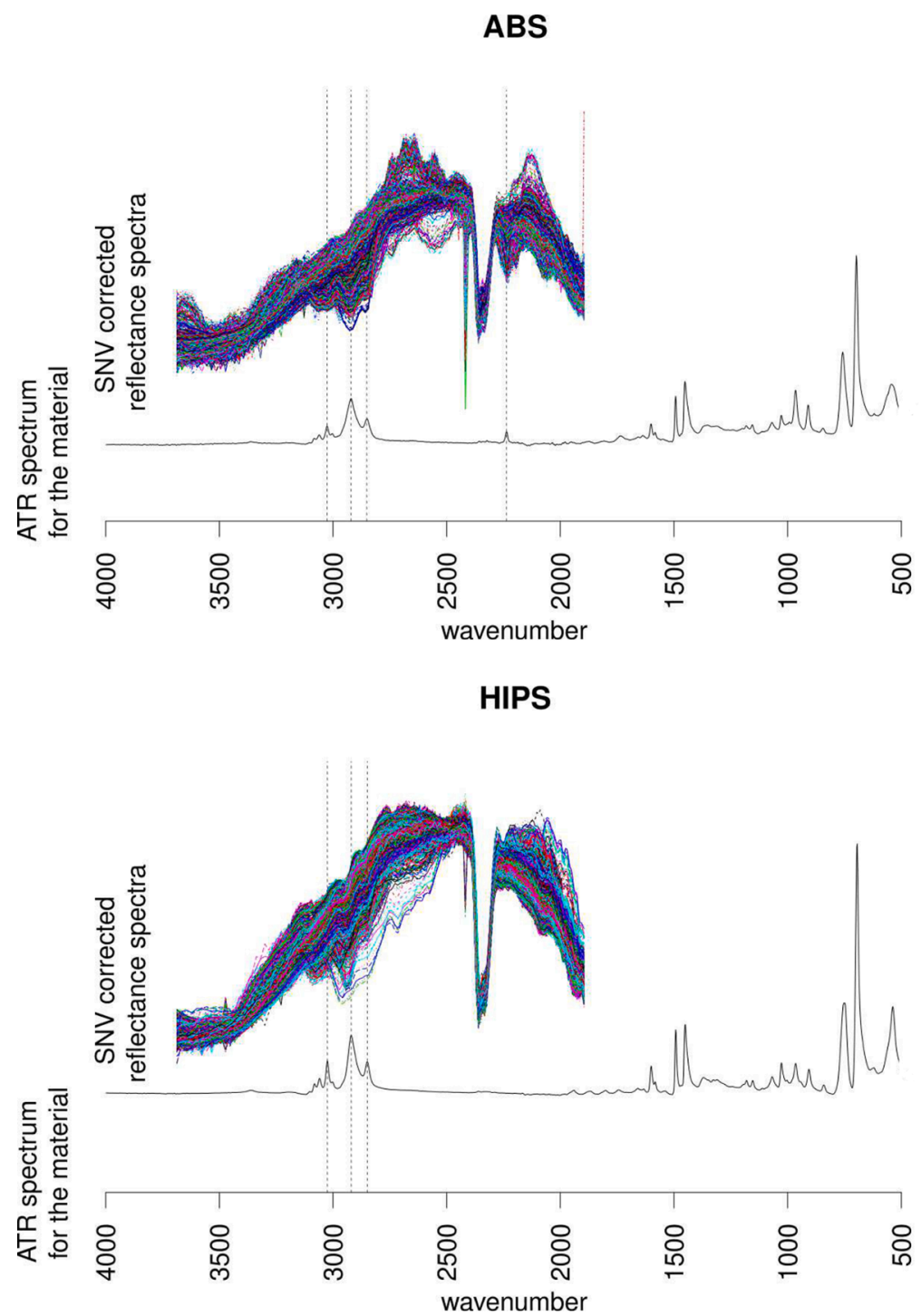

Fig. 3. Spectra of the four plastic types compared with their corresponding ATR reference spectrum. 
PE

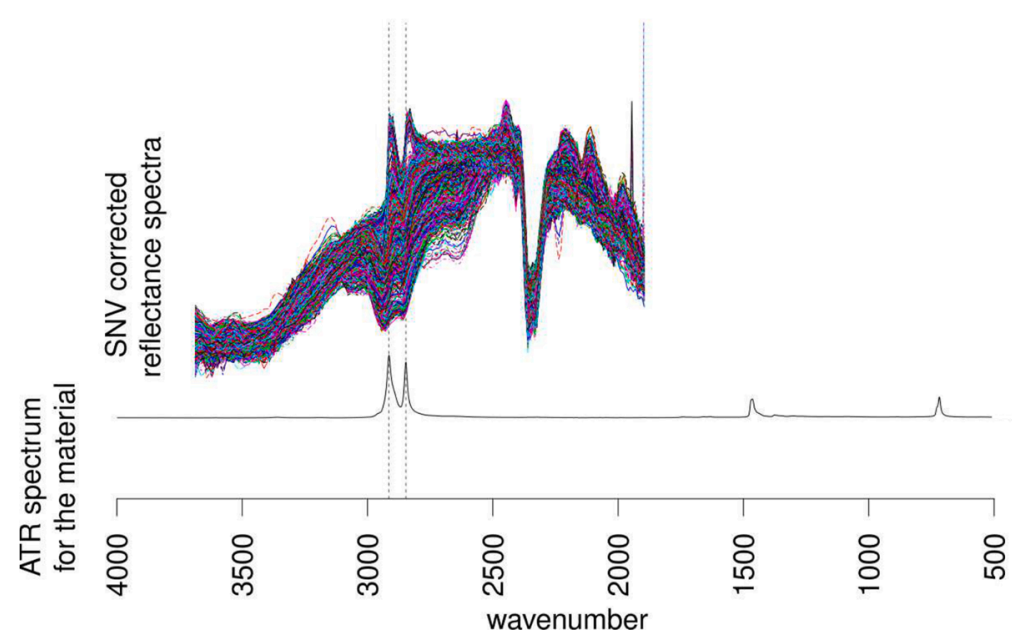

PP

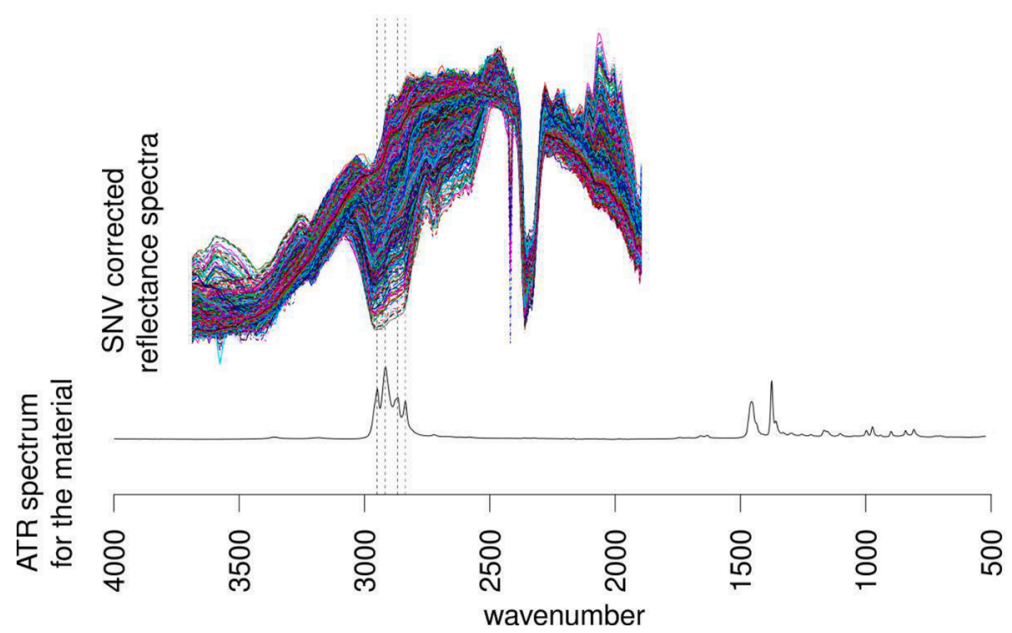

Fig. 3. (continued).

- There are also some classification algorithms outputting mass functions with more focal elements. For example, ECLAIR (Evidential CLAssification of incomplete data via Imprecise Relabelling) proposed in Jacquin et al. (2019) provides a belief function-based version of any probabilistic classifier. This algorithm showed competitive results on spectra obtained for WEEE plastics by MIR technology in an industrial context. ECLAIR can assign masses to all subsets $A$ of $\Omega$.
Once one of the above classifiers has been trained, it is applied to all pixels of $f r$. Then $k$ mass functions representing the information concerning each pixel $m\left(. \mid s_{1}\right), \cdots, m\left(. \mid s_{k}\right)$ are obtained.

\subsubsection{Fragment classification: Combining pixels information}

In order to synthesize the mass functions provided by each pixel for the fragment $f r$, it is necessary to combine all the mass functions $m\left(. \mid s_{1}\right)$, $\cdots, m\left(. \mid s_{k}\right)$. The most popular combining procedure used in the belief

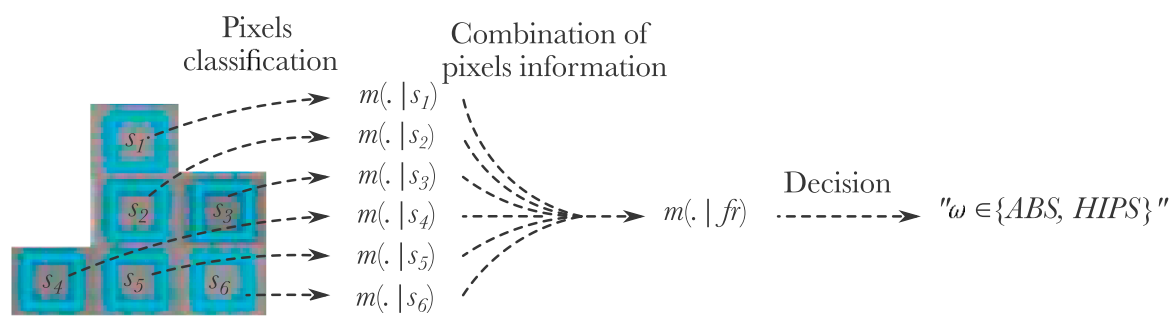

Example of a plastic fragment $f r$

Fig. 4. Schema of the proposed procedure on a example. The notation $m(. \mid x)$ is explained in more details in 2.3.1. 
Table 1

Table of losses $L_{\beta}(A, B)$.

\begin{tabular}{|c|c|c|c|c|c|c|c|c|}
\hline & $\mathrm{B} / \mathrm{A}$ & $A B S$ & HIPS & $P E$ & $P P$ & Styrenic & Polyolefin & Ignorance \\
\hline \multirow[t]{3}{*}{$\beta=0.1$} & Styrenic & 0.010 & 0.010 & 1 & 1 & 0 & 1 & 0.498 \\
\hline & $\{H I P S, P E\}$ & 1 & 0.010 & 0.010 & 1 & 0.500 & 0.500 & 0.498 \\
\hline & $\{A B S, H I P S, P E\}$ & 0.019 & 0.01 & 0.019 & 1 & 0.005 & 0.502 & 0.248 \\
\hline \multirow[t]{3}{*}{$\beta=3$} & Styrenic & 0.474 & 0.474 & 1 & 1 & 0 & 1 & 0.091 \\
\hline & $\{H I P S, P E\}$ & 1 & 0.474 & 0.474 & 1 & 0.50 & 0.500 & 0.091 \\
\hline & $\{A B S, H I P S, P E\}$ & 0.643 & 0.643 & 0.643 & 1 & 0.31 & 0.655 & 0.032 \\
\hline
\end{tabular}

Table 2

$\operatorname{Risk}_{\beta}(A)$ for $\beta=0.1$ and $\beta=3$.

\begin{tabular}{|c|c|c|c|c|c|c|c|}
\hline A & $A B S$ & HIPS & $P E$ & $P P$ & Styrenic & Polyolefin & Ignorance \\
\hline $\operatorname{Risk}_{0.1}(A)$ & 0.603 & 0.107 & 0.604 & 1 & 0.349 & 0.8 & 0.573 \\
\hline $\operatorname{Risk}_{3}(A)$ & 0.742 & 0.432 & 0.789 & 1 & 0.227 & 0.8 & 0.133 \\
\hline
\end{tabular}

function framework is Dempster combination (conjunctive combination) which assumes that all sources of information are independent, trustworthy and that there is no conflict between them. Although the source of information for each pixel can reasonably be considered as independent, this combination does not seem appropriate in our case since the information obtained for some pixels severely affected by uncertainty might not always provide information consistent with the information coming from others. Another common combination is the disjunctive combination rule, which also assumes that sources of information are independent but is appropriate for scn is reliable (Denœux, 2008). For a fragment $f r$ composed, for example, by two pixels $s_{1}$ and $s_{2}$, the disjunctive combination of $m\left(. \mid s_{1}\right)$ and $m\left(. \mid s_{2}\right)$ is defined for a subset of plastic types $A$ by:

$$
m_{1()_{2}}(A \mid f r)=\left\{\begin{array}{l}
\sum_{A=B \cup C} m\left(B \mid s_{1}\right) m\left(C \mid s_{2}\right) \quad \text { if } A \neq \emptyset, \\
0
\end{array}\right.
$$

In the case of more pixels $(k>2)$ the disjunctive combination of all the pixel masses is computed using the associative property of the rule.

Example 2. Consider the following example with $k=2$, such that:

for pixel $s_{1}: m\left(\{H I P S\} \mid s_{1}\right)=0.4, \quad m\left(\{A B S, H I P S\} \mid s_{1}\right)=0.6$,

for pixel $s_{2}: m\left(\{A B S\} \mid s_{2}\right)=0.2, m\left(\{P E, H I P S\} \mid s_{2}\right)=0.8$.

Then

$$
\begin{aligned}
m_{1(\bigcup)}(\{A B S, H I P S\} \mid f r) & =0.2, \\
m_{1}()_{2}(\{H I P S, P E\} \mid f r) & =0.32, \\
m_{1(\bigcup)}(\{A B S, H I P S, P E\} \mid f r) & =0.48 .
\end{aligned}
$$

It should be noted that this combination can assign masses to new subsets such as $\{A B S, H I P S, P E\}$ in this example.

Alternatively, the mass functions can also be combined by taking the mean operator, for a subset of plastic types $A$ :

$m(A \mid f r)=\frac{\sum_{i=1}^{k} m\left(A \mid s_{i}\right)}{k}$.
Taking example 2 , the mean combination of $m\left(. \mid s_{1}\right)$ and $m\left(. \mid s_{2}\right)$ gives:

$$
\begin{aligned}
m(\{H I P S\} \mid f r) & =0.2, \quad m(\{A B S, H I P S\} \mid f r) & =0.3, \\
m(\{A B S\} \mid f r) & =0.1, \quad m(\{P E, H I P S\} \mid f r) & =0.4 .
\end{aligned}
$$

There is a justification for using both disjunctive and mean combination in the application. However, mean combination does not produce new focal elements and is less computationally complex. The mean combination rule was therefore chosen in this work to merge the information about all pixels.

\subsection{Decision}

Once the information has been collected and synthesised for $f r$, it needs to be processed via a decision phase in order to return the final output. Classically, in the case of a standard classification problem, the decision phase would aim at returning the most suitable material-type depending on probabilities and on certain specificities of the problem expressed in the form of a loss function $L$. This loss function classically defined on $\Omega \times \Omega$ quantifies the loss incurred when assigning a certain material-type when the true material-type is either different or the same. For example, having $L(A B S, H I P S)=0.8$ would mean that the loss incurred when mistaking a real HIPS fragment for a $A B S$ fragment is penalized by a loss of 0.8 . Using a loss function enables different types of errors to be penalised differently. This is particularly useful in our application since different types of errors correspond to different types of impurities that may not have the same consequences. The most

Table 4

\begin{tabular}{|c|c|c|c|c|c|c|c|c|}
\hline & Train & & & & Test & & & \\
\hline & $A B S$ & HIPS & $P E$ & $P P$ & $A B S$ & HIPS & $P E$ & $P P$ \\
\hline Number of fragments & 35 & 35 & 35 & 35 & 35 & 35 & 35 & 35 \\
\hline Total number of pixels & 2934 & 2895 & 2975 & 2943 & 2887 & 2960 & 2882 & 2889 \\
\hline
\end{tabular}

Evaluation of cautious classification algorithms obtained with the proposed procedure.

\begin{tabular}{llll}
\hline Classifier providing the mass functions & relevance & reliability & $F_{1}$ \\
\hline Probabilistic version of SVM & $\mathbf{0 . 9 1 1}$ & 0.925 & 0.918 \\
Probabilistic version of $k$-nn & 0.910 & 0.930 & $\mathbf{0 . 9 2 0}$ \\
Belief function based $k$-nn & 0.821 & $\mathbf{0 . 9 5 4}$ & 0.883 \\
ECLAIR version SVM & 0.896 & 0.930 & 0.913 \\
ECLAIR version $k$-nn & 0.897 & 0.940 & 0.918 \\
\hline
\end{tabular}

Table 3

Database. 


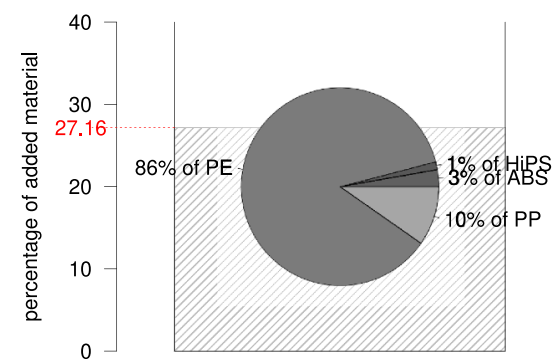

Fig. 5. Example of representation for a container output result.

common loss function, called the $0-1$ loss function, simply gives a maximal loss 1 for mistakes and minimal loss 0 when the assigned material matches the true material. After defining a loss function, the decision classically relies on expected loss minimisation also referred to as risk minimisation. For each candidate material $a \in \Omega$ to be the real material-type of $f r$, the risk, i.e. expected loss, is computed according to the posterior probability $p(. \mid f r)$ in the following way:

$\operatorname{Risk}(a)=\sum_{b \in \Omega} p(b \mid f r) L(a, b)$,

then the chosen material is the one with the least risk. For example, considering the $0-1$ loss function and the probabilities $p(A B S \mid f r)=0.8$ and $p(H I P S \mid f r)=0.2$, leads to

$$
\begin{gathered}
\operatorname{Risk}(A B S)=p(A B S \mid f r) L(A B S, A B S)+p(H I P S \mid f r) L(A B S, H I P S) \\
=0.8 \times 0+0.2 \times 1
\end{gathered}
$$$$
=0.8 \text {. }
$$

Similarly, Risk $(H I P S)=0.2$. In fact, risk minimisation considering $0-1$ loss always amounts to choosing the material having the highest probability. In order to produce a cautious output, i.e. returning a set $A$, it is also necessary to define loss functions for subset outputs. Therefore, returning to the framework of the belief function, where the information is $m(. \mid f r)$ instead of $p(. \mid f r)$, it is possible to adapt standard risk minimisation in order to produce cautious outputs. One adaption was proposed in Jacquin et al. (2019). The main idea is to consider a loss function defined by considering both truth and candidate as subsets. For example, $L(\Omega,\{A B S, H I P S\})=0.4$ means that the loss incurred by assigning $\Omega$, i.e. outputting the ignorance, when the "real" material-type is Styrenic (see example 1) is 0.4 . A possible loss for a subset can be constructed by taking the maximal loss of its elements. We can also consider a loss function that assigns losses to subsets without taking into account the elements it contains. Such a loss function based on an evaluation criterion is presented in the next subsection. In this case, for each candidate output subset $A$, the risk is defined by:

$\operatorname{Risk}(A)=\sum_{B \subseteq \Omega} m(B \mid f r) L(A, B)$.

\subsection{Evaluation of cautious outputs}

In this subsection, the evaluation of cautious output is discussed. The classical evaluation is based on the equality between the truth and the prediction. However, the evaluation of cautiousness needs to compare the truth and a predicted subset output. This has been the object of several works (Yang et al., 2016; Zaffalon et al., 2012). In order to illustrate the difficulty of evaluating a cautious classifier, the argument presented by Zaffalon et al. (2012) is as follows (adapted for our context). Let us consider the classification task with only two material-types $\{A B S, H I P S\}$. Consider the following two extreme classifiers: one is a random classifier, i.e. it randomly returns a single material-type in $\{A B S, H I P S\}$, the other is a vacuous classifier, i.e. it

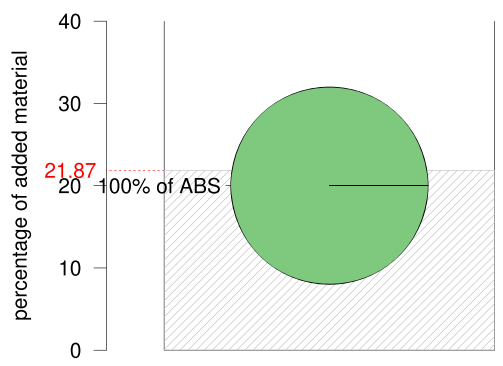

(a) Container dedicated to $A B S$

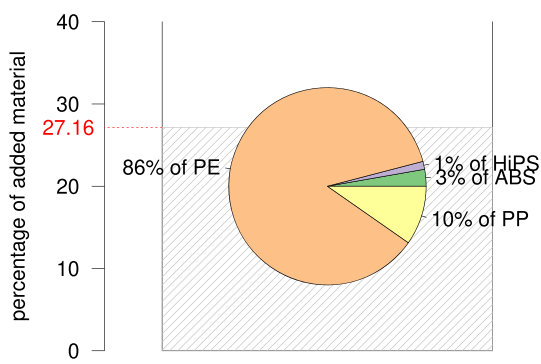

(c) Container dedicated to $P E$

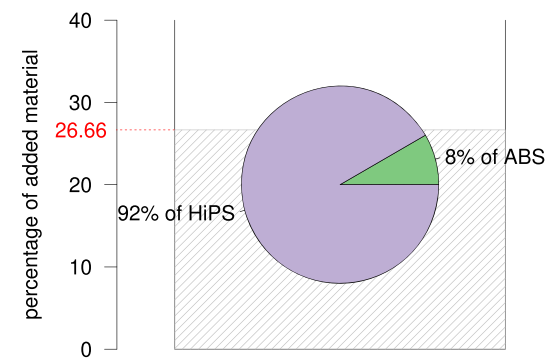

(b) Container dedicated to $H I P S$

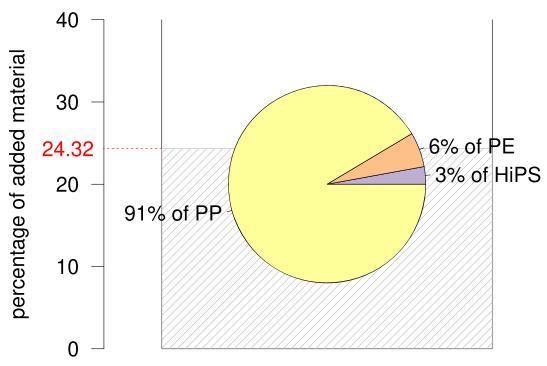

(d) Container dedicated to $P P$

Fig. 6. Sorting results with the conventional voting procedure. 


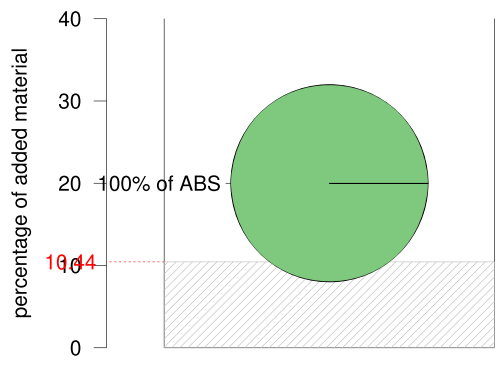

(a) Container dedicated to $A B S$

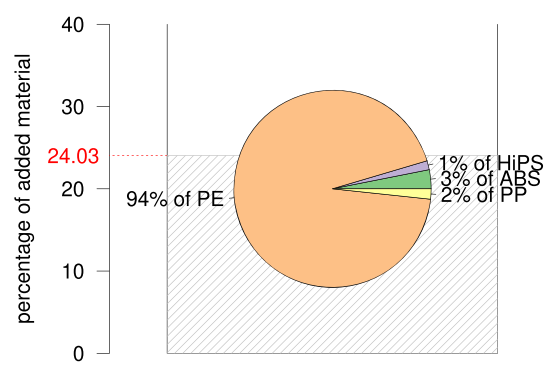

(c) Container dedicated to $P E$

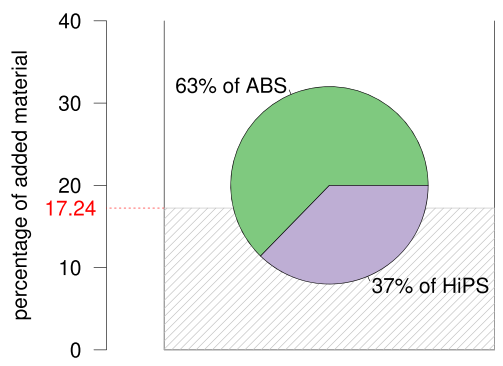

(e) Container dedicated to Styrenic

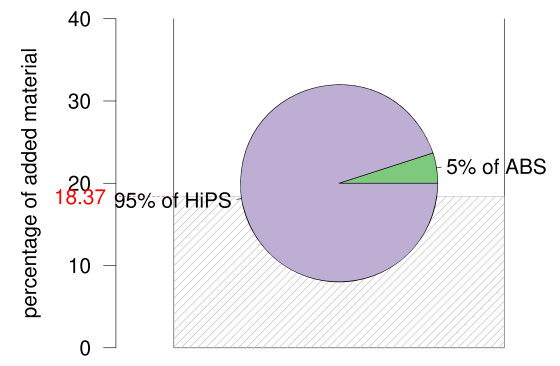

(b) Container dedicated to $H I P S$

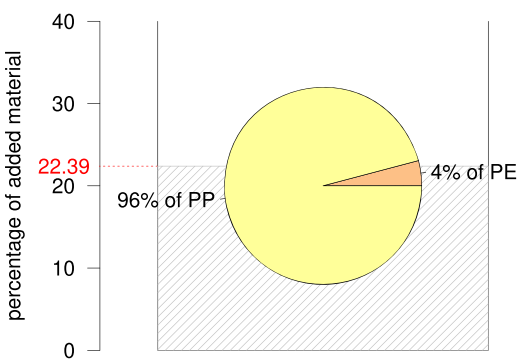

(d) Container dedicated to $P P$

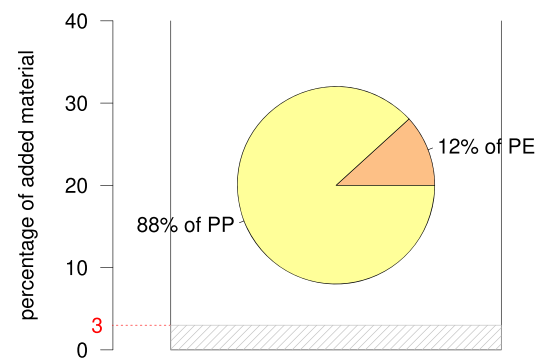

(f) Container dedicated to Polyolefin

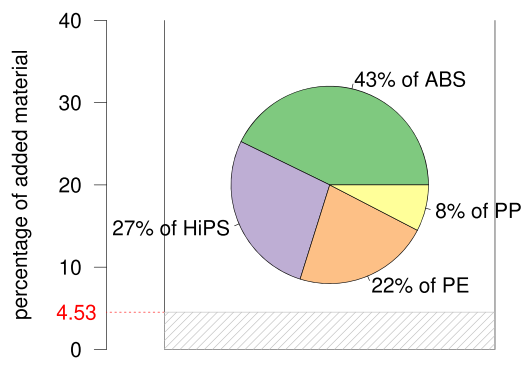

(g) Container dedicated to Ignorance

Fig. 7. Sorting results with the proposed cautious procedure.

always returns the set $\{A B S, H I P S\}$. Both classifiers are unaware of the real material-types but they have opposite ways of managing their ignorance. How should these classifiers be evaluated? On average, the random classifier is wrong half of the time on a balanced testing database thus the expected reward would be $1 / 2$. The reward of the vacuous classifier is more difficult to assess because it never makes real mistakes, but it does not provide any relevant information either. However, it is arguable that the behaviour of the vacuous classifier is preferable because it expresses its inability, thus this classifier should be rewarded more than $1 / 2$. In order to implement this distinction, many evaluation 
measures for cautious classification have been proposed in the literature: the F-measure (Coz et al., 2009), the utility Discounted accuracy (Zaffalon et al., 2012) or the p-discounted cost (Yang et al., 2016), etc. In this article, only the generalized evaluation measures of those proposed in Coz et al. (2009) are presented since they seem sufficient to understand the main challenges when evaluating a cautious output. To avoid confusion with classical measures of precision and recall, we refer to the measures proposed in $\mathrm{Coz}$ et al. (2009) as measures of reliability and relevance. Furthermore, the definitions are given in the general case, considering both truth and classification output as subsets, respectively denoted $B$ and $A$. The reliability corresponds to the proportion of the true material-types $(B)$ included in the cautious output $(A)$ :

$\operatorname{reliability}(A, B)=\frac{|A \cap B|}{|B|}$,

where $|A|$ denotes the number of elements in $A$. We denote by $1_{A}$ the indicator function defined on $\Omega$ having the value 1 for all elements of $A$ and the value 0 for all elements of $\Omega$ not in $A$. When the true materialtype $B$ is a singleton $B=\{\omega\}$, we have reliability $(A,\{\omega\})=1_{A}(\omega)$. On the other hand, the relevance is defined as the proportion of outputted material-types $(A)$ that are true $(B)$ :

relevance $(A, B)=\frac{|A \cap B|}{|A|}$.

Similarly, when the true material-type $B$ is a singleton $B=\{\omega\}$, we have relevance $(A,\{\omega\})=1_{A}(\omega) /|A|$.

Example 3. reliability $(\{A B S, H I P S\}, A B S)=1$ and relevance $(\{A B S, H I P S\}, A B S)=1 / 2$.

Note that the measures of reliability and relevance defined above concern a single fragment. Those measures are defined for a set of fragments as the average of the measures obtained for each fragment. The presented measures enable a different aspect of the cautious output to be appreciated: for reliability, the true material-type needs to belong to the cautious output; for relevance, the cautious output also has to be as small as possible. Thus reliability tends to promote output with bigger subsets $A$ because the bigger $A$ is the higher the chance of it containing $\omega$. Conversely, reliability tends to promote smaller subsets. The main challenge of cautious classification is to find a compromise between reliability and relevance. This compromise varies according to the situation, and can be evaluated using the $F_{\beta}$ measure (for cautious classification) defined in Coz et al. (2009):

$F_{\beta}(A, \omega)=\frac{\left(1+\beta^{2}\right) \text { relevance }(A, \omega) \cdot \text { reliability }(A, \omega)}{\beta^{2} \text { relevance }(A, \omega)+\text { reliability }(A, \omega)}$,

with a parameter $\beta>0$ that controls the degree of cautiousness. With small values of $\beta$, the output relevance is emphasised, i.e. the size of the output should be reasonable, while high values of $\beta$ emphasising reliability, i.e. the true material should be included in the output set. Continuing with the example 3 ; for a small $\beta$, e.g. $\beta=0.1, F_{0.1}(\{A B S$, $H I P S\}, A B S)=0.5024876$ is obtained and for a higher $\beta$, e.g. $\beta=3$, $F_{3}(\{A B S, H I P S\}, A B S)=0.9090909$.

The $F_{\beta}$ evaluation measure can also be useful for defining a loss function for the risk minimisation of cautious outputs mentioned in the previous section. This was first done by Coz et al. (2009) and adapted in Jacquin et al. (2019) to the framework of belief functions.

Considering the true material subset $B$ instead of a single true material $\omega$, the following generalisation of $F_{\beta}$ for subsets $A$ and $B$ is obtained:

$F_{\beta}(A, B)=\frac{\left(1+\beta^{2}\right)|A \cap B|}{\beta^{2}|B|+|A|}$.

In this article, the loss function used to minimise the risk defined in the Eq. (4) is defined as $L(A, B)=1-F_{\beta}(A, B)$.
In order to illustrate the influence of $\beta$ in the decision phase, consider the example 2:

$\begin{aligned} m(\{H I P S\} \mid f r) & =0.2, \quad m(\{A B S, H I P S\} \mid f r) & =0.3, \\ m(\{A B S\} \mid f r) & =0.1, \quad m(\{P E, H I P S\} \mid f r) & =0.4 .\end{aligned}$

Table 1 shows losses obtained with $\beta=0.1$ and $\beta=3$. It should be mentioned that only the losses $L_{\beta}(A, B)$ for meaningful outputs $A$, i.e. that could correspond to valuable containers, and for sets $B$ involved in the risk calculation are presented. Note also that $\{A B S, H I P S\}$ is referred to as Styrenic, $\{P E, P P\}$ as Polyolefin and $\Omega$ as Ignorance.

Table 1 shows that the lack of relevance is more penalised for $\beta=0.1$ than for $\beta=3$ (the losses are higher with $\beta=0.1$ when the cardinality of $A$ is bigger than the cardinality of $B$ ). Using the table of losses, the risk of candidate outputs can be computed for the two values of $\beta$. The resulting risks are shown in Table 2 (minimal risk values are shown in bold).

Finally, as shown in Table 2 , choosing $\beta=0.1$, would lead the cautious classifier to return HIPS as material-type for the fragment $f r$ which means that the fragment fr would be blown into a container dedicated to HIPS. Similarly, choosing $\beta=3$ would lead to blowing $\mathrm{fr}$ into a container dedicated to more than one material. Finally, depending on the application, the decision-maker can choose $\beta$ according to the required reliability and relevance.

\section{Results and discussion}

In this section, experiments are conducted to show the benefits of coupling MIR imaging and cautious classification for optimizing recycling. First, the database is presented and some results of several cautious algorithms (all based on the proposed procedure) are shown. Then an example of cautious sorting is compared with a more conventional sorting procedure. Ultimately the results and some possible adaptations are discussed.

The experiments were carried out on the 280 plastic fragments provided by SUEZ company. After being recorded by the camera, each plastics fragment region of interest (roi) was selected manually to obtain $3 \mathrm{D}$ reflection image as shown in Fig. 2. One half of the fragments were used as training and the other half for testing. The division of fragments by types can be found in Table 3. Note that the total number of pixels varies depending on the different categories since each fragment is composed by a different number of pixels according to its size (the average number of pixels composing a fragment in this database is 83).

First of all, some cautious classification algorithms obtained by the proposed procedure are evaluated. As mentioned in Section 2.3.1, in its pixel classification phase the proposed cautious procedure uses an algorithm able to return a mass function. Many choices of algorithms are practicable, thus for ease of exposition, the algorithms already presented 2.3.1, i.e. probabilistic classifiers, the belief function-based version of the $k$-nearest neighbour classifier and ECLAIR classifiers, are used in this illustration. As probabilistic and ECLAIR classifiers are generic algorithms, it was also necessary to choose specific algorithms. The versions of $k$-nearest neighbour $(k-n n)$ and Support Vector Machine (SVM) were selected because they are efficient algorithms. Finally, the algorithms considered are: two probabilistic versions of $k$-nn and SVM classifiers, the belief function-based version of $k$-nearest neighbour classifier, and two ECLAIR versions of $k$-nn and SVM classifiers. In order to reduce the complexity and enhance the performances, Linear Discriminant Analysis (LDA) was applied as a pre-processing operation. These different classification algorithms were used for the "pixel classification" phase and the loss function defined in 2.5 was used for setting the risk minimisation with $\beta=1$, meaning that relevance and reliability are considered equally important in the decision phase. The cautious classifications results are presented in Table 4.

Table 4 shows that the choice of algorithm has an influence on cautious classification performances. The results show that none of the classifiers are best in terms of both relevance and reliability. In the next 
application, ECLAIR version of SVM was selected because it finds a good compromise between relevance and reliability and because SVM is more appropriate than $k$-nn for real-time industrial applications. Although it was not shown here, to simplify the reading of Table 4 , the $\beta$ parameter also has an influence on the performances (see previous section) and should be optimized according to the required compromise between relevance and reliability. In the rest of the paper, the proposed cautious sorting procedure is compared with a more conventional industrial sorting procedure. The cautious procedure was run using the ECLAIR version of SVM and setting $\beta=1.6$, which could be optimized using cross validation. The possible output (dedicated containers) were fixed to $A B S, H I P S, P E, P P$, Styrenic, Polyolefin and Ignorance. Regarding the conventional sorting procedure, the voting approach presented in Section 1, taking a standard SVM classifier to assign each pixel a single plastic type was applied. In this procedure, the possible dedicated containers are $A B S, H I P S, P E, P P$. The two procedures are compared by looking at the final real composition of the containers in terms of quantity, i.e. the fill rate of containers, and composition.

The sorting results obtained by the voting approach are shown in Fig. 6. Each sub-figure should be read as follows: the quantity of added material is calculated in terms of percentage of the total sorted plastics and displayed in red on the y-axis (hatched lines represent the filling). Note that in the ideal case where all the testing fragments are perfectly sorted, the fill rate in the precise containers would be approximately $25 \%$ (see Table 3), more precisely it would be $24.85 \%$ for $A B S, 25.48 \%$ for HIPS, $24.81 \%$ for $P E$ and $24.87 \%$ for $P P$. The pie plots represent the real composition of the container after the sorting procedure. For example, the explanation for sub-Fig. $6 \mathrm{c}$ also showed in Fig. 5: the filling rate of this container is $27.16 \%$ of total sorted plastics. Its composition is mainly $P E(86 \%)$ but also impurities, $10 \%$ of $P P, 3 \%$ of $A B S$ and $1 \%$ of HIPS. The sorting results obtained with the proposed cautious procedure involve two more containers that are chemically meaningful: Styrenic, Polyolefin, and an additional container dedicated to Ignorance. The results are shown in Fig. 7.

Comparing Figs. 6 and 7, one observes that the quantities of sorted material in the precise container, $A B S, H I P S, P E$ and $P P$ declined in the cautious procedure compared to the voting procedure. Indeed comparing the sub-Figs. $6 \mathrm{a}$ with 7 a one notes that the percentage of material in the container dedicated to $A B S$ has decreased by - $52.26 \%$. Similarly $-30.07 \%$ for HIPS, $-11.52 \%$ for $P E,-9.35 \%$ for PP. On the other hand, the purity of containers was enhanced with the cautious procedure: $+2 \%$ for HIPS, $+8 \%$ for $P E$ and $+6 \%$ for $P P$. Although there is a serious decline in quantity with the cautious procedure, it enhances the purity rate by a few percent, which could enable sorting quality standards to be reached. Note that this sorting quality has a great impact on the quality of the future recycled material. For example, a previous work (Perrin et al., 2016) studies the values of maximal impurity tolerance within HIPS, it states that without compatibilizing agents, maximal tolerance of $A B S$ within HIPS can only be up to $4 \%$. This level is almost obtained with the proposed procedure. Moreover, with the proposed approach, the containers Styrenic and Polyolefin do not contain any impurity, which is also a valuable outcome. Indeed these sorted materials could be used for less demanding recycling use or could be integrated in a sorting procedure based on several sorting stages. There are some points that may be important to discuss concerning the proposed cautious sorting approach. Firstly, the compromise between relevance and reliability should be considered carefully. When sorting errors do not have serious consequences, output relevance should predominate in order to maximise the quantity of precisely sorted material. When errors have serious consequences, one should keep in mind that although ensuring high reliability would lead to an increase in sorting quality, it will also naturally decline the quantity of sorted material. Industrialist designing sorting algorithms will need to define their own loss function to penalise the different types of errors, rather than the generic loss function used in this article. Secondly, the cautious procedure takes its full meaning when it is integrated in a real-time sorting of material fragments under constraints on compositions (see recent works dealing with problem of real-time sorting of fragments under constraints on containers compositions, Jacquin et al. (2020b), Jacquin et al. (2020a)). Considering such an issue, cautious sorting algorithms are able to track the evolution of knowledge about a container composition in real time and make inferences about requirements on impurity constraints for optimizing the final sorting result.

Finally, on a more technical level, the proposed cautious sorting approach assumes a device able to blow fragments into more containers than in conventional sorting procedures. This might require the technical adaptation of sorting devices. However, note that the proposed procedure is flexible concerning the number of considered containers and is practicable even with fewer containers.

\section{Conclusion}

Artificial intelligence, more precisely machine learning, is a promising solution for attaining minimum recovery targets of WEEE and has already convinced many industrialists. However industrial-scale technologies to discriminate between plastics provide uncertain/imprecise information that compromises the sorting task. Sorting errors seriously affect the quality of the recycled material. In this article a cautious sorting algorithm is proposed to increase the purity of sorted materials based on MIR imaging. Note that this cautious sorting algorithm is completely transposable to other context for sorting of other materials. The given sorting procedure is based on a belief function framework to better represent the uncertainty of the data and provide more cautious sorting by enabling plastic fragments to be blown into containers dedicated to more than a single material-type. The procedure was compared to a more conventional procedure that does not enable cautious outputs. It was observed that cautiousness enhances sorting purity, which is essential for optimizing the quality of secondary raw materials. Naturally, cautiousness also decreases the quantity of precisely sorted material but this can be controlled by defining a loss function suitable for the considered application. The first perspective of this work could be to improve the proposed approach by taking into account morphological aspects of the fragments. Indeed, the pixels on the edges are often less reliable and can disturb the classification process. By discounting the beliefs coming from pixels on the edges of plastic fragments, information could be better represented and taken into account in the cautious output, thus the quality of sorted material could be further improved. A second perspective is the integration of the proposed approach in real industrial sorting conditions, which implies computational complexity issues in cases where a large number of fragments are considered.

On a more general note, the work presented in this article is part of a wider trend that aims at managing waste using artificial intelligence (AI). Digitization through the implementation of artificial intelligence is a driving force for innovation activities in many sectors of green technologies, such as renewable energy or sustainable mobility. It is a tool that can considerably improve the quality of sorting through careful decision-making depending on the type of WEEE plastics supplied by the Suez firm, leading to the recovery of efficient second-life raw materials that are economically affordable and sustainable. This work is a first step towards this future perspective of sorting/identifying recycled materials 2.0 .

\section{CRediT authorship contribution statement}

Lucie Jacquin: Conceptualization, Formal analysis, Investigation, Validation, Writing - original draft. Abdelhak Imoussaten: Conceptualization, Formal analysis, Methodology, Validation, Writing - review \& editing. François Trousset: Conceptualization, Methodology, Writing review \& editing. Didier Perrin: Project administration, Supervision, Validation, Writing - review \& editing. Jacky Montmain: Project administration, Supervision, Validation, Writing - review \& editing. 


\section{Declaration of Competing Interest}

The authors declare that they have no known competing financial interests or personal relationships that could have appeared to influence the work reported in this paper.

\section{Acknowledgements}

The authors would like to acknowledge the support of the companies Pellenc st and Suez S.A. for the collecting of spectra obtained for WEEE plastics by MIR technology in an industrial context.

\section{References}

, 2012Article 11 recovery targets, directive 2012/19/eu of the european parliament and of the council of 4 july 2012 on waste electrical and electronic equipment (WEEE). Official Journal of the European Union, 2012.

Bae, J.-S., Oh, S.-K., Pedrycz, W., Fu, Z., 2019. Design of fuzzy radial basis function neural network classifier based on information data preprocessing for recycling black plastic wastes: comparative studies of ATR FT-IR and raman spectroscopy. Applied Intelligence 49 (3), 929-949.

Beigbeder, J., Perrin, D., Mascaro, J.-F., Lopez-Cuesta, J.-M., 2013. Study of the physicochemical properties of recycled polymers from waste electrical and electronic equipment (WEEE) sorted by high resolution near infrared devices. Resour. Conserv. Recycl. 78, 105-114.

Chow, C., 1970. On optimum recognition error and reject tradeoff. IEEE Trans. Inf. Theory 16 (1), 41-46.

Coz, J.J.d., Díez, J., Bahamonde, A., 2009. Learning nondeterministic classifiers. Journal of Machine Learning Research 10 (Oct), 2273-2293.

Cucchiella, F., D’Adamo, I., Koh, S.L., Rosa, P., 2015. Recycling of WEEEs: an economic assessment of present and future e-waste streams. Renewable Sustainable Energy Rev. 51, 263-272.

Dempster, A.P., 1967. Upper and lower probability inferences based on a sample from a finite univariate population. Biometrika 54 (3-4), 515-528.

Denoeux, T., 1995. A k-nearest neighbor classification rule based on dempster-shafer theory. IEEE Trans Syst Man Cybern 25 (5), 804-813.

Denoeux, T., 2000. A neural network classifier based on dempster-shafer theory. IEEE Transactions on Systems, Man, and Cybernetics-Part A: Systems and Humans 30 (2), $131-150$.

Denœux, T., 2008. Conjunctive and disjunctive combination of belief functions induced by nondistinct bodies of evidence. Artif Intell 172 (2-3), 234-264.

Denoeux, T., 2008. A K-nearest neighbor classification rule based on dempster-shafer theory. Classic works of the Dempster-Shafer theory of belief functions. Springer, pp. 737-760.

Dubois, D., Prade, H., 2012. Possibility Theory. Springer.

Dunn, J.C., 1973. A Fuzzy Relative of the ISODATA Process and Its Use in Detecting Compact Well-Separated Clusters. Journal of Cybernetics 3 (3), 32-57 https://doi. org/10.1080/01969727308546046.

Ha, T.M., 1997. The optimum class-selective rejection rule. IEEE Trans Pattern Anal Mach Intell 19 (6), 608-615.

Jacquin, L., Imoussaten, A., Destercke, S., 2020. Handling mixture optimisation problem using cautious predictions and belief functions. International Conference on Information Processing and Management of Uncertainty in Knowledge-Based Systems. Springer.

Jacquin, L., Imoussaten, A., Destercke, S., Trousset, F., Montmain, J., Perrin, D., 2020. Manipulating focal sets on the unit simplex: application to plastic sorting. Submitted to FUZZ-IEEE. IEEE.
Jacquin, L., Imoussaten, A., Trousset, F., Montmain, J., Perrin, D., 2019. Evidential classification of incomplete data via imprecise relabelling: application to plastic sorting. International Conference on Scalable Uncertainty Management. Springer, pp. 122-135.

Kassouf, A., Maalouly, J., Rutledge, D.N., Chebib, H., Ducruet, V., 2014. Rapid discrimination of plastic packaging materials using MIR spectroscopy coupled with independent components analysis (ICA). Waste Manage. (Oxford) 34 (11), 2131-2138.

Keller, J.M., Gray, M.R., Givens, J.A., 1985. A fuzzy k-nearest neighbor algorithm. IEEE Trans Syst Man Cybern (4), 580-585.

Kruse, R., 2008. Fuzzy neural network. Scholarpedia 3 (11), 6043

Lachaize, M. Le Hégarat-Mascle, S., Aldea, E., Maitrot, A., Reynaud, R., 2018. Evidential split-and-merge: application to object-based image analysis. Int. J. Approximate Reasoning 103, 303-319.

Lorente, D., Aleixos, N., Gómez-Sanchis, J., Cubero, S., García-Navarrete, O.L., Blasco, J., 2012. Recent advances and applications of hyperspectral imaging for fruit and vegetable quality assessment. Food Bioproc Tech 5 (4), 1121-1142.

Matarazzo, A., Tuccio, G., Teodoro, G., Failla, F., Giuffrida, V.A., 2019. Mass balance as green economic and sustainable management in WEEE sector. Energy Procedia 157, 1377-1384.

Nguyen, V.-L., Destercke, S., Masson, M.-H., Hüllermeier, E., 2018. Reliable multi-class classification based on pairwise epistemic and aleatoric uncertainty. International Joint Conference on Artificial Intelligence.

Blanch-Perez-del Notario, C., Saeysb, W., Lambrechtsc, A., 2019. Hyperspectral imaging for textile sorting in the visible-near infrared range. Journal of Spectral Imaging 8.

Ongondo, F.O., Williams, I.D., Cherrett, T.J., 2011. How are WEEE doing? a global review of the management of electrical and electronic wastes. Waste Manage. (Oxford) 31 (4), 714-730.

Paclık, P., Duin, R.P., 2005. Designing multi-modal classifiers of spectra: a study on industrial sorting application. 2nd Spectral Imaging workshop, Villach, Austria.

Paclik, P., Leitner, R., Duin, R.P., 2006. A study on design of object sorting algorithms in the industrial application using hyperspectral imaging. Journal of Real-Time Image Processing 1 (2), 101-108.

Perrin, D., Mantaux, O., Ienny, P., Léger, R., Dumon, M., Lopez-Cuesta, J.-M., 2016. Influence of impurities on the performances of hips recycled from waste electric and electronic equipment (WEEE). Waste Manage. (Oxford) 56, 438-445.

Roh, S.-B., Oh, S.-K., Park, E.-K., Choi, W.Z., 2017. Identification of black plastics realized with the aid of raman spectroscopy and fuzzy radial basis function neural networks classifier. J. Mater. Cycles Waste Manage. 19 (3), 1093-1105.

Rozenstein, O., Puckrin, E., Adamowski, J., 2017. Development of a new approach based on midwave infrared spectroscopy for post-consumer black plastic waste sorting in the recycling industry. Waste Manage. (Oxford) 68, 38-44.

Shafer, G., 1976. A Mathematical Theory of Evidence, 42. Princeton University Press.

Signoret, C., Caro-Bretelle, A.-S., Lopez-Cuesta, J.-M., Ienny, P., Perrin, D., 2019. MIR spectral characterization of plastic to enable discrimination in an industrial recycling context: i. specific case of styrenic polymers. Waste Manage. (Oxford) 95, 513-525.

Signoret, C., Caro-Bretelle, A.-S., Lopez-Cuesta, J.-M., Ienny, P., Perrin, D., 2019. MIR spectral characterization of plastic to enable discrimination in an industrial recycling context: II. specific case of polyolefins. Waste Manage. (Oxford) 98, 160-172.

Signoret, C., Caro-Bretelle, A.-S., Lopez-Cuesta, J.-M., Ienny, P., Perrin, D., 2019. MIR spectral characterization of plastic to enable discrimination in an industrial recycling context: II. specific case of polyolefins. Waste Manage. (Oxford) 98, 160-172.

Smets, P., Kennes, R., 1994. The transferable belief model. Artif Intell 66 (2), 191-234.

Walley, P., 2000. Towards a unified theory of imprecise probability. Int. J. Approximate Reasoning 24 (2-3), 125-148.

Yang, G., Destercke, S., Masson, M.-H., 2016. The costs of indeterminacy: how to determine them? IEEE Trans Cybern 47 (12), 4316-4327.

Zadeh, L.A., 1978. Fuzzy sets as a basis for a theory of possibility. Fuzzy Sets Syst. 1 (1), 3-28.

Zaffalon, M., Corani, G., Mauá, D., 2012. Evaluating credal classifiers by utilitydiscounted predictive accuracy. Int. J. Approximate Reasoning 53 (8), 1282-1301. 